\title{
Associação entre a prática de atividade física em diferentes domínios e o uso de insulina em adultos e idosos com diabetes no Brasil
}

\author{
Association between the practice of physical activity of different \\ types and the use of insulin in adult and elderly diabetics in Brazil
}

Anne Ribeiro Streb (http://orcid.org/0000-0001-9195-4210) ${ }^{1}$
Larissa dos Santos Leonel (http://orcid.org/0000-0002-5778-055X) ${ }^{2}$
Caroline Soares da Silva (https://orcid.org/0000-0001-6053-0159) ${ }^{3}$
Robert Passos da Silva (https://orcid.org/0000-0002-9456-1997) ${ }^{1}$
Giovani Firpo Del Duca (https://orcid.org/0000-0003-0893-2032) ${ }^{3}$
${ }^{1}$ Programa de Pós-

Graduação em Educação Física, Universidade Federal de Santa Catarina (UFSC). R. Roberto Sampaio Gonzaga s/n, Trindade. 88040-900 Florianópolis SC Brasil.tina.anne@ hotmail.com

${ }^{2}$ Campus Universitário Reitor João David Ferreira Lima. UFSC. Florianópolis SC Brasil.

${ }^{3}$ Graduação em Educação Física, UFSC. Florianópolis SC Brasil.

\begin{abstract}
The scope of this paper was to verify the association between the practice of physical activity of different types and the use of insulin in adults and the elderly. This is a cross-sectional population-based study with data from the survey entitled Surveillance of risk factors and protection for chronic diseases by telephone (VIGITEL 2013). The sample consisted of individuals aged $\geq 18$ years, living in the 27 Brazilian capitals and diagnosed with diabetes. The practice of physical activity in commuting, the home, leisure, work and the use of insulin were self-reported. Binary logistic regression was used in crude and adjusted analyses for sociodemographic factors. Of the 4,593 subjects with diabetes, insulin use was reported by $16.2 \%$ and $25.5 \%$ of adults and the elderly, respectively. The practice of physical activity in the home (OR: 0.61, 95\%CI: 0.40; 0.94) and at work (OR: 0.38, 95\% CI: $0.18 ; 0.78)$ were associated with the lowest use of insulin among the elderly. There was a tendency to reduce insulin use as the number of physically active domains increased among the elderly ( $p=0.003)$. The conclusion drawn was that physical activity practiced alone or in a cumulative way in different domains was associated with lower insulin use among the elderly.

Key words Medication, Diabetes mellitus, Motor activity, Cross-sectional studies
\end{abstract}

Resumo Objetivou-se verificar a associação entre a prática de atividade física em diferentes domínios e o uso de insulina em adultos e idosos. Trata-se de um estudo transversal de base populacional com dados do inquérito Vigilância de fatores de risco e proteção para doenças crônicas por Inquérito Telefônico (VIGITEL 2013). A amostra foi composta por indivíduos com idade $\geq 18$ anos, residentes nas 27 capitais do Brasil e com diagnóstico de diabetes. A prática de atividade física no deslocamento, domicílio, lazer e trabalho, e o uso de insulina foram por autorrelato. Empregouse regressão logística binária em análises bruta e ajustada para fatores sociodemográficos. Dos 4.593 indivíduos com diabetes, uso de insulina foi relatado por $16,2 \%$ e $25,5 \%$ dos adultos e idosos, respectivamente. A prática de atividade física no domicílio (RO: 0,61; IC95\%: 0,40; 0,94) no trabalho (RO: 0,38; IC95\%: 0,18; 0,78) estiveram associadas ao menor uso de insulina em idosos. Houve tendência de redução do uso de insulina à medida que se aumentou a quantidade de dominios fisicamente ativos em idosos ( $p=0,003)$. Conclui-se que, em idosos, a atividade física praticada de forma isolada ou de forma acumulada em diferentes domínios teve associação com o menor uso de insulina.

Palavras-chave Medicamento, Diabetes mellitus, Atividade motora, Estudos transversais 


\section{Introdução}

A diabetes mellitus consiste em um distúrbio hormonal caracterizado por hiperglicemia persistente decorrente da deficiência na produção e/ ou na ação da insulina ${ }^{1}$. Atualmente a diabetes é uma importante causa de morbidade e mortalidade, que tem estimativas globais para cerca de 592 milhões de pessoas com a doença no ano de $2035^{2}$. Como forma de controle da diabetes, tanto da 1 como da 2, utiliza-se de estratégias que incluem planejamento nutricional, uso de fármacos, doses de insulina, monitoração da glicemia e prática regular de exercícios físicos ${ }^{3}$. O tradicional tratamento medicamentoso aos pacientes que possuem deficiência na produção de insulina, ou seja, no diabetes tipo 1, é o uso exógeno de insulina injetável; Já aos indivíduos com diabetes do tipo 2, onde há deficiência na ação da insulina caracterizada pela resistência à tal, preconiza-se o uso de medicações orais, entretanto, em casos de não responsividade as altas dosagens e combinações de hiperglicemiantes, costuma-se associarse a utilização de insulina ${ }^{4}$. Seu uso é justificado devido ao declínio fisiopatológico progressivo das células pancreáticas ou ao alto grau de descompensação metabólica provenientes de outras doenças e/ou agravamento da própria diabetes ${ }^{1}$.

Já o tratamento não medicamentoso, que inclui mudanças relacionadas ao estilo de vida, especialmente na inclusão da prática regular de atividade física, têm sido fortemente recomendadas, evitando consequências deletérias advindas do uso de medicamentos ${ }^{5}$. Nos seus diferentes domínios (deslocamento, domicílio, lazer e trabalho), a atividade física se apresenta como um fator de proteção para a diabetes, em decorrência de um maior gasto energético semanal em atividade física ${ }^{6,7}$. Um estudo que relacionou os domínios da atividade física e a diabetes mellitus apontou que a prática no lazer e a acumulada, isto é, o somatório de todos os domínios são as melhores preditoras da ausência desta doença, enquanto os demais domínios apresentam menor capacidade para tal ${ }^{8}$.

Ao confrontar a prática de atividade física em diferentes domínios e a diabetes, consistentes evidências apontam que esta prática ocasiona redução da hemoglobina glicada e aumento de sensibilidade à insulina ${ }^{3,9}$. No entanto, a relação do nível habitual de atividade física total e em domínios e o uso de medicamentos, em especial a insulina, ainda carece de mais investigações. Dentre os poucos estudos que exploram a temática, uma pesquisa realizada com brasilei- ros abordando a prática de atividade física total concluiu que, comparados aos sujeitos fisicamente ativos, aqueles inativos utilizaram 23\% mais medicamentos ${ }^{10}$. Quando analisado o nível de atividade física somente no domínio lazer, a prática com duração de pelo menos 150 minutos semanais apresentou redução significativa no uso de medicamentos ${ }^{11}$. Já um estudo realizado com suecos não encontrou associações significativas com o uso de medicamentos e a atividade física no lazer ${ }^{12}$. A pouca literatura se demonstra conflitante em seus resultados com o uso de medicamentos em geral e os níveis de atividade física em diferentes domínios. Tendo em vista o processo de transição epidemiológica das últimas décadas, torna-se relevante investigar a relação entre os comportamentos relacionados ao estilo de vida individual e a possível redução do uso de medicamentos para o controle/tratamento da diabetes mellitus. Diante deste cenário, objetivou-se verificar a associação entre uso de insulina para o tratamento da diabetes mellitus com indicadores de atividade física em diferentes domínios em adultos e idosos no Brasil.

\section{Métodos}

Este estudo se caracteriza como transversal, de base populacional e consiste em uma análise secundária de dados do inquérito nacional Vigilância de fatores de risco e proteção para doenças crônicas por Inquérito Telefônico (VIGITEL) do ano de 2013. Teve-se como população alvo indivíduos com idade $\geq 18$ anos, residentes nas 27 capitais do Brasil. Especificamente neste estudo, foram incluídos todos os indivíduos que relataram ter diagnóstico médico de diabetes.

O cálculo amostral foi realizado de forma independente para cada localidade, estabelecendo um tamanho amostral mínimo de 2.000 indivíduos em cada cidade. O processo de seleção consistiu em estratificar as linhas telefônicas reconhecidas das elegíveis, no qual foram sorteadas por código de endereçamento postal e de forma sistemática, com, no mínimo, 5.000 números por capital. Foram identificadas 74.005 linhas elegíveis para levantamento. As estimativas obtidas foram ajustadas à população brasileira, com o uso do peso pós-estratificação considerando o sexo, a faixa etária e o nível de escolaridade. O peso pós-estratificação de cada indivíduo da amostra foi calculado pelo método rake. Maiores detalhes sobre o processo de seleção de linhas telefónicas, bem como, o processo de amostragem 
encontra-se disponível no relatório do VIGITEL $2013^{13}$.

Todas as variáveis utilizadas foram obtidas a partir da aplicação de um questionário validado por entrevista telefônica ${ }^{14}$. A variável dependente deste estudo foi o uso de insulina para tratamento de diabetes. Para isso, considerou-se como usuário de insulina aqueles indivíduos que responderam "sim" à questão: "Atualmente, o(a) Sr(a) está usando insulina para controlar a diabetes?”. A variável independente do estudo foi a prática de atividade física nos seus diferentes domínios. A categorização do domínio lazer foi dada a partir das questões "Na última semana, o(a) Sr(a) praticou algum tipo de exercício físico ou esporte?”. Com relação ao domínio deslocamento, as questões que forneceram informações sobre foram: "Para ir ou voltar ao seu trabalho, faz algum trajeto a pé ou de bicicleta?", "Atualmente, o(a) Sr(a) está frequentando algum curso/escola ou leva alguém em algum curso/escola?", "Para ir ou voltar a este curso ou escola, faz algum trajeto a pé ou de bicicleta?”. Para o domínio domicílio, utilizou-se a questão "Quem costuma fazer a faxina da sua casa?" e para o trabalho, "No seu trabalho, o(a) $\operatorname{Sr}($ a) anda bastante a pé?", "No seu trabalho, o(a) $\mathrm{Sr}(\mathrm{a})$ carrega peso ou faz outra atividade pesada?". Considerou-se como fisicamente ativo todos os sujeitos com resposta afirmativa nas respectivas questões de cada domínio investigado.

Para análise dos dados, utilizou-se o programa Stata ${ }^{\circledR}$, versão 13.0, levando em consideração os fatores de ponderação da amostragem complexa. Inicialmente, foram apresentadas as frequências absolutas e relativas (valores apresentados de acordo com a ponderação por amostragem complexa) das variáveis utilizadas. Para testar a associação dos domínios de atividade física e o uso de insulina empregou-se a regressão logística binária estratificada em adultos (de 18 a 59 anos) e idosos ( $\geq 60$ anos). Essas análises foram ajustadas conforme modelo hierárquico, sendo que o sexo, a idade, a situação conjugal e a cor da pele foram incluídas no primeiro nível e a escolaridade, no segundo. Para esta modelagem estatística, foi adotada a estratégia de seleção para trás e um nível crítico de $\mathrm{p} \leq 0,20$ para permanência no modelo, com intuito de controle de confusão.

Para testar as associações individuais entre a atividade física em domínios e ainda o acúmulo de 2 ou mais destes com a ocorrência do uso de insulina, utilizou-se a regressão logística binária ajustada para variáveis sociodemográficas, considerando estatisticamente significantes os valores de $\mathrm{p} \leq 0,05$. Os resultados foram expressos como razões de odds (RO) e intervalos de confiança de 95\% (IC95\%).

O VIGITEL 2013 obteve aprovação da Comissão Nacional de Ética em Pesquisa para Seres Humanos do Ministério da Saúde. O consentimento livre e esclarecido foi obtido oralmente no momento do contato telefônico com os entrevistados.

\section{Resultados}

Dos 4.593 indivíduos com diabetes investigados, 50,5\% eram adultos. A maioria da amostra de adultos e idosos eram do sexo feminino (52,8\%; $60,0 \%$, respectivamente) e viviam com companheiro(a): 55,4\% adultos; 55,3\% idosos. A média de idade foi de $36,2 \pm 11,2$ anos em adultos e, $69,4 \pm 13,3$ anos em idosos. A utilização de insulina foi relatada por $16,2 \%$ dos adultos e $25,5 \%$ dos idosos. Mais detalhes sobre a caracterização da amostra estão descritos na Tabela 1 .

Na Tabela 2 estão descritos os resultados da associação entre a prática de atividade física em diferentes domínios e o uso de insulina para o tratamento de diabetes mellitus, estratificados por idade. É possível observar que, independentemente da idade, sexo, região, cor da pele, estado civil e escolaridade, a prática de atividades físicas no domicílio (RO: 0,61; IC95\%: 0,40; 0,94) e trabalho (RO: 0,38; IC95\%: 0,18; 0,78) esteve associada ao menor o uso de insulina em idosos.

As Figuras 1 e 2 levam em consideração a prática de atividade física acumulada nos diferentes domínios em adultos e idosos, respectivamente. Em idosos, é possível observar que há tendência de redução do uso de insulina à medida que se aumenta a quantidade de domínios ativos.

\section{Discussão}

O presente artigo buscou investigar a associação entre o uso de insulina para o tratamento de diabetes com indicadores de atividade física em diferentes domínios em adultos e idosos no Brasil. Destaca-se como principal resultado a menor probabilidade do uso de insulina quando ocorre a prática de atividade física nos domínios trabalho e domicílio em idosos. Nesse sentido, Almeida et al. $^{6}$ identificaram que mulheres são mais ativas no domicílio quando comparadas aos homens, possivelmente devido ao arranjo cultural associar estas tarefas ao sexo feminino ${ }^{6}$. No nosso estudo, o sexo feminino foi prevalente, o 
Tabela 1. Descrição das características dos adultos e idosos diabéticos residentes nas capitais brasileiras ( $\mathrm{n}$ = 4.593). Brasil, 2013 .

\begin{tabular}{|c|c|c|}
\hline Variável & $\begin{array}{c}\text { Adultos n } \\
\left(\%^{\mathrm{a}}\right)\end{array}$ & $\begin{array}{c}\text { Idosos n } \\
\left(\%^{\mathrm{a}}\right)\end{array}$ \\
\hline \multicolumn{3}{|l|}{ Sexo } \\
\hline Masculino & $686(47,2)$ & $962(40,0)$ \\
\hline Feminino & $1.055(52,8)$ & $1.890(60,0)$ \\
\hline \multicolumn{3}{|l|}{ Situação Conjugal } \\
\hline Sem companheiro (a) & $657(34,6)$ & $1.426(44,7)$ \\
\hline Com companheiro (a) & $1.065(55,4)$ & $1.385(55,3)$ \\
\hline \multicolumn{3}{|c|}{ Cor da pele } \\
\hline Branca & $661(41,9)$ & $1.277(57,8)$ \\
\hline Preta & $161(13,9)$ & $230(10,9)$ \\
\hline Parda & $661(41,3)$ & $745(26,2)$ \\
\hline Outras & $58(2,9)$ & $112(5,1)$ \\
\hline \multicolumn{3}{|l|}{ Regiões } \\
\hline Centro-oeste & $250(9,0)$ & $465(9,5)$ \\
\hline Nordeste & $602(24,9)$ & $955(22,0)$ \\
\hline Norte & $433(8,3)$ & $499(6,5)$ \\
\hline Sudeste & $283(50,3)$ & $512(53,7)$ \\
\hline Sul & $173(7,5)$ & $421(8,3)$ \\
\hline \multicolumn{3}{|l|}{ Escolaridade (anos) } \\
\hline 0 a 8 & $624(55,1)$ & $1.616(76,2)$ \\
\hline 9 a 11 & $660(29,4)$ & $645(14,8)$ \\
\hline$\geq 12$ & $442(15,5)$ & $503(9,0)$ \\
\hline \multicolumn{3}{|l|}{$\begin{array}{l}\text { Uso de insulina por } \\
\text { diabéticos }\end{array}$} \\
\hline Não & $1.423(83,8)$ & $2.229(74,5)$ \\
\hline Sim & $316(16,2)$ & $617(25,5)$ \\
\hline \multicolumn{3}{|l|}{ AF deslocamento } \\
\hline Não & $1.302(70,2)$ & $2.584(89,4)$ \\
\hline Sim & $426(29,8)$ & $242(10,6)$ \\
\hline \multicolumn{3}{|l|}{ AF domicílio } \\
\hline Não & $989(57,2)$ & $2.017(71,4)$ \\
\hline Sim & $747(42,8)$ & $824(28,6)$ \\
\hline \multicolumn{3}{|l|}{ AF lazer } \\
\hline Não & $1.021(62,2)$ & $1.777(67,6)$ \\
\hline Sim & $720(37,8)$ & $1.075(32,4)$ \\
\hline \multicolumn{3}{|l|}{ AF trabalho } \\
\hline Não & $1.145(62,0)$ & $2.535(87,9)$ \\
\hline Sim & $596(38,0)$ & $317(12,1)$ \\
\hline
\end{tabular}

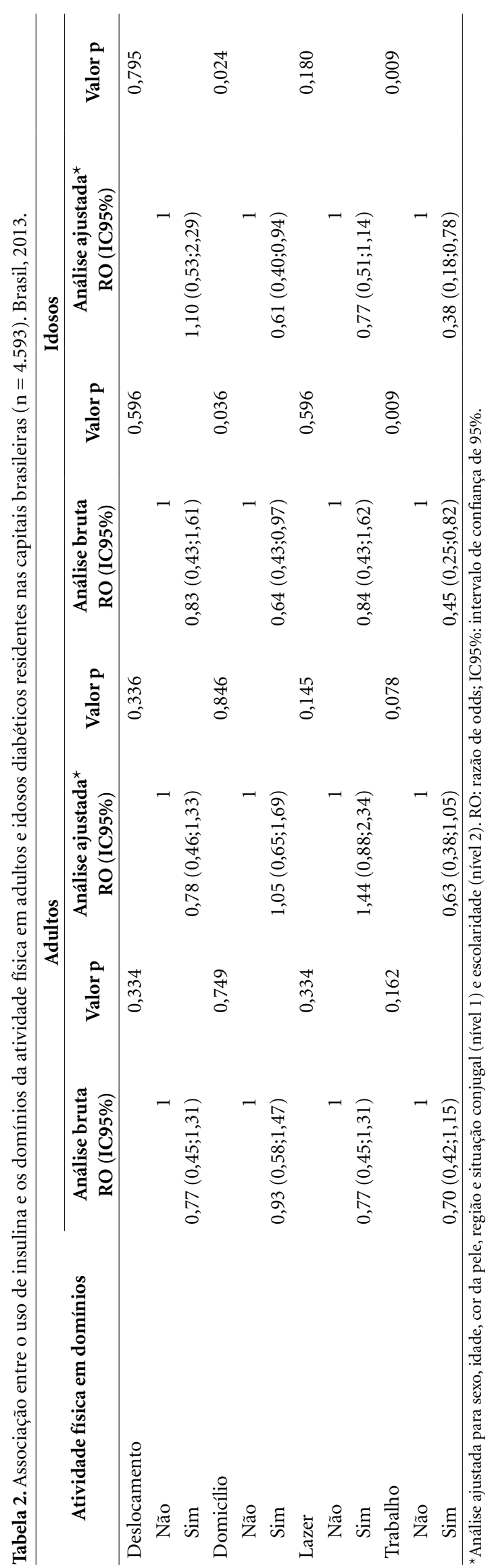




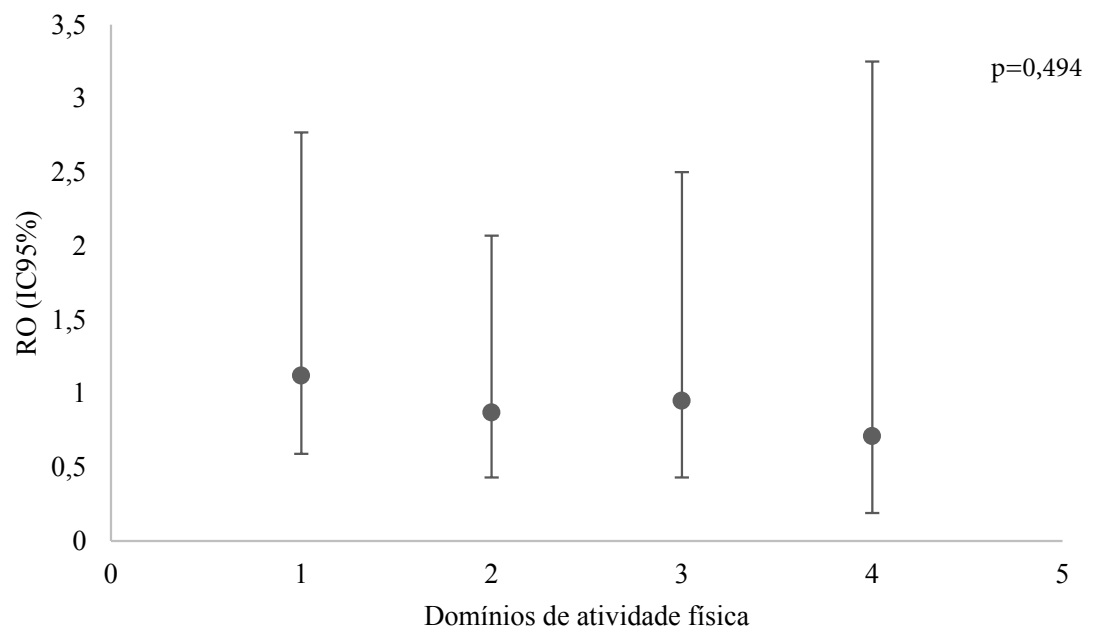

Figura 1. Associação entre a prática de atividade física em somatório de domínios e o uso de insulina em adultos ( $\mathrm{n}=1.741)$. Brasil, 2013.

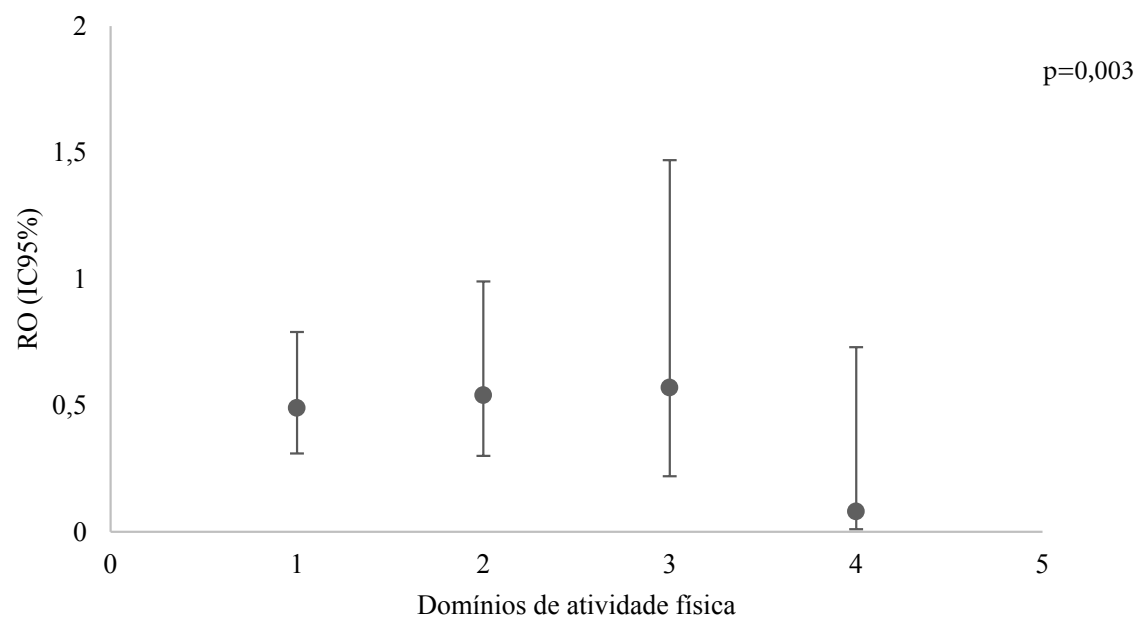

Figura 2. Associação entre a prática de atividade física em somatório de domínios e o uso de insulina em idosos ( $\mathrm{n}=2.852)$. Brasil, 2013.

que deve ser ponderado ao se analisar os achados. Além disso, foi evidenciado em uma metanálise que o turno de trabalho, para homens, implica em maior risco de desenvolver diabetes ${ }^{15}$. O trabalho noturno foi associado ao aumento de chances à tal doença. Inclusive, também costuma ser associado a menores níveis de atividade física e maior tempo em comportamento sedentário ${ }^{16}$.

Dentre os diabéticos que foram entrevistados pelo VIGITEL no ano de 2013, 25,5\% dos idosos utilizavam insulina como forma de tratamento para esta doença. A ocorrência da diabe- 
tes cresceu consideravelmente nos últimos anos, principalmente nos países de renda média como o Brasil ${ }^{17}$. Esse fato pode ser explicado por conta do aumento da expectativa de vida da população e, em contrapartida, pelo estilo de vida marcado pela adoção de comportamentos de risco. Destaca-se ainda que a diabetes tem se desenvolvido cada vez mais em adultos jovens, o que reforça a importância de mudanças relacionadas as atitudes da população ${ }^{1}$.

A prática de atividade física, evidenciada neste estudo nos domínios do trabalho e domicílio, parece ser coadjuvante na redução do uso de insulina para o tratamento de diabetes. Embora, não se tenha encontrado associações significativas da prática de atividades físicas no lazer com a redução do uso de insulina tanto para adultos, quanto para idosos, há evidências de tal prática está correlacionada com a redução do risco de diabetes, principalmente quando realizada em intensidades elevadas ${ }^{18}$. Segundo Costa Junior et al. ${ }^{19}$, a prática de exercício físico no lazer pode implicar na redução da depuração de insulina e aumentar a sensibilidade para tal.

A não obtenção de associações com a prática de atividade física em domínios e a utilização de insulina aos adultos pode parcialmente ser explicado pelo fato de que, a insulina costuma ser considerada como fármaco para o tratamento da diabetes tipo 1, comumente desvendada na adolescência, gerando dependência à sua utilização endógena, autonomamente à prática de atividades físicas. Já, para aqueles diagnosticados com diabetes tipo 2, seu uso costuma ser prescrito quando a utilização de medicamentos orais já passou por interações, aumento de dosagens e não consegue mais ser efetiva. Retratando assim, uma progressão da doença, evidenciada ao passar dos anos, agravada em idosos ${ }^{4}$. Mesmo assim, com base no coletivo de investigações, a Associação Americana de Diabetes recomenda que exercícios físicos sejam prescritos para os indivíduos com diabetes como parte fundamental do controle glicêmico e da saúde geral, com precauções específicas de acordo com o tipo de diabetes, idade, atividade realizada e presença de complicações relacionadas ${ }^{3}$.

Um estudo realizado com idosos na cidade de Campinas-SP demonstrou que 21,7\% desta população possui diabetes e sua maioria faz combinações de medicamentos hipoglicemiantes orais com a insulina, além disso, relatam baixa prática de atividade física no lazer. Porém, 62,7\% não referiram complicações devido a doença e dizem conseguir realizar suas atividades diárias básicas e domésticas ${ }^{20}$. Isso indica que, apesar da baixa prática de atividade física no lazer decorrentes de fatores intrínsecos, eles realizam suas atividades domésticas com êxito. Mesmo sabendo que esta população superestima tais atividades, no entanto, é plausível considerar a contribuição destes momentos para a composição das atividades diárias. É relevante destacar que, não apenas a atividade física no tempo livre, mas as atividades físicas no trabalho e no deslocamento também são importantes componentes do estilo de vida ativo em indivíduos diabéticos, contribuindo para minimizar os efeitos deletérios ocasionados pela doença ${ }^{6}$.

Com relação ao somatório de atividades nos domínios, ficou evidenciado nos idosos que, à medida que se acresce comportamentos ativos ao longo do dia, o risco do uso de insulina parece sofrer uma redução gradual. Devido ao aumento da sensibilidade à insulina periférica promovido pela prática de atividade física, há depuração de insulina e melhor captação pelo GLUT-4, acarretando na melhora do quadro de diabetes e, consequentemente, na redução do uso de insulina para o tratamento. Evidências apontam uma relação direta com a frequência semanal, intensidade e o tipo de atividade, apresentando efeito dose-resposta ${ }^{21-23}$.

O presente estudo, por se tratar de uma coleta de dados com delineamento transversal, impossibilitou estabelecer uma relação de causa-efeito dos resultados, mas possibilitará um entendimento da associação entre domínios da atividade física com o uso de insulina, forma de tratamento, pouco investigada, de uma das doenças altamente prevalentes na população brasileira. Ainda, a medida do uso de insulina impossibilita a obtenção de informações quanto à dosagem e posologia, que consideramos relevante, que poderia implicar em novos resultados e discussões, ademais, não se sabe qual é o tipo de diabetes que estas pessoas relataram ter. Todavia, deve ser considerado a originalidade da temática, que se propõe a avaliar em âmbito populacional, o uso de medicamentos. Conforme os dados da Pesquisa Nacional de Saúde do ano de 2013, no Brasil, a proporção de pessoas de 18 anos ou mais de idade que referiram diagnóstico médico de diabetes e tomaram medicamento para a diabetes ou usaram insulina nas duas últimas semanas anteriores à data da pesquisa é de $80,2 \%{ }^{24}$. Um número expressivo que resulta em elevados investimentos do sistema público de saúde, podendo ser otimizado com a prática de atividade física em diferentes domínios. Ademais, as direções dos presentes resultados, quando significativas, foram em prol da atividade física, isolada ou acumulada nos distintos domínios. Ao avaliar aqui um dos critérios 
de $\mathrm{Hill}^{25}$, que é a consistência, somadas às outras evidências literárias relativas percebe-se que a atividade física possui um efeito protetor às doenças crônicas, dentre elas a diabetes. Além disso, pode também guardar relação não apenas com a doença em si, mas também com o tratamento por uso de medicamentos.

Conclui-se, portanto, que a prática de atividade física nos domínios trabalho e domicílio, resultam na redução de chance de utilizar insuli- na como forma de tratamento para a diabetes em idosos, com percentuais de reduções à medida que se agregam práticas ativas em mais domínios nesta mesma população. Recomenda-se que estudos futuros empreguem delineamentos longitudinais para se entender com mais propriedade a relação causal entre a prática de atividade física e o uso de medicamentos para o tratamento de diabetes em distintas populações.

\section{Colaboradores}

AR Streb, LS Leonel e GFD Del Duca participaram de todas as etapas do estudo. CS Silva, RP Silva contribuiu com a redação do artigo e análise dos resultados. Todos os autores aprovaram a versão final a ser publicada e se declaram responsáveis pelo manuscrito na garantia da exatidão e integridade de qualquer parte da obra. 


\section{Referências}

1. Oliveira JEP, Montenegro Junior RM, Vencio S, organizadores. Diretrizes da Sociedade Brasileira de Diabetes 2017-2018. São Paulo: Editora Clannad; 2017.

2. Guariguata L, Whiting DR, Hambleton I, Beagley J, Linnenkamp U, Shaw JE. Global estimates of diabetes prevalence for 2013 and projections for 2035. Diabetes Res Clin Pract 2014; 103(2):137-149.

3. American Heart Association. Living Healthy with Diabetes [Internet]. 2018 [acessado 2018 Apr 18]. Disponível em: http://www.heart.org/HEARTORG/ Conditions/More/Diabetes/PreventionTreatment ofDiabetes/Living-Healthy-with-Diabetes_UCM_ 313880_Article.jsp\#.WtawFYjwZPZ

4. Elk N, Iwuchukwu OF. Using Personalized Medicine in the Management of Diabetes Mellitus. Pharmacother J Hum Pharmacol Drug Ther 2017; 37(9):1131-1149.

5. Sigal RJ, Armstrong MJ, Bacon SL, Boulé NG, Dasgupta K, Kenny GP, Riddell MC. Physical Activity and Diabetes. Can J Diabetes 2018; 42(Supl. 1):S54-S63.

6. Almeida LAB, Pitanga FJG, Freitas MM, Pitanga CPS, Dantas EHM, Beck CC. Gasto calórico dos diferentes domínios de atividade física como preditor da ausência de diabetes em adultos. Rev Bras Med Esporte 2012; 18(1):17-21.

7. Hu G, Qiao Q, Silventoinen K, Eriksson JG, Jousilahti P, Lindström J, Valle TT, Nissinen A, Tuomilehto J. Occupational, commuting, and leisure-time physical activity in relation to risk for Type 2 diabetes in middle-aged Finnish men and women. Diabetologia 2003; 46:322-329.

8. Pitanga FJ, Almeida LA, Freitas MM, Pitanga CP, Beck CC. Padrões de atividade física em diferentes domínios e ausência de diabetes em adultos. Motricidade 2010; 6(1):5-17.

9. Zuo H, Shi Z, Yuan B, Dai Y, Hu G, Wu G, Hussain A. Interaction between physical activity and sleep duration in relation to insulin resistance among non-diabetic Chinese adults. BMC Public Health 2012; 12:47.

10. Bertoldi AD, Hallal PC, Barros AJ. Physical activity and medicine use: evidence from a population-based study. BMC Public Health 2006; 6:24.

11. Garcia LMT, Salvador EP, Sá TH, Florindo AA. Associação entre prática de atividade física no tempo de lazer e medicação permanente em adultos de uma região de baixo nível socioeconômico. Rev Bras Cineantropometria Desempenho Hum 2014; 16:371.

12. Bardel A, Wallander MA, Svärdsudd K. Reported current use of prescription drugs and some of its determinants among 35 to 65 -year-old women in mid -Sweden: A population-based study. J Clin Epidemiol 2000; 53(6):637-643.

13. Brasil. Ministério da Saúde (MS). Vigitel Brasil 2013: Vigilância de fatores de risco e proteção para doenças crônicas por inquérito telefônico. Brasília: MS; 2013.

14. Monteiro CA, Moura EC, Jaime PC, Lucca A, Florindo AA, Figueiredo ICR, Bernal R, Silva NN. Monitoramento de fatores de risco para doenças crônicas por entrevistas telefônicas. Rev Saúde Pública 2005; 39(1):47-57.

15. Gan Y, Yang C, Tong X, Sun H, Cong Y, Yin X, Li L, Cao S, Dong X, Gong Y, Shi O, Deng J, Bi H, Lu Z. Shift work and diabetes mellitus: a meta-analysis of observational studies. Occup Environ Med 2015; 72(1):72-78
16. Silva Garcez A, Canuto R, Paniz VMV, Olinto BA, Macagnan J, Henn RL, Pattussi MP, Olinto MTA. Association between work shift and the practice of physical activity among workers of a poultry processing plant in Southern Brazil. Nutr Hosp 2015; 31(5):2174-2181.

17. Stopa SR, César CLG, Segri NJ, Goldbaum M, Guimarães VMV, Alves MCGP, Barros MBA. Self-reported diabetes in older people: comparison of prevalences and control measures. Rev Saúde Pública 2014; 48(4):554-662.

18. Huai P, Han H, Reilly KH, Guo X, Zhang J, Xu A. Leisure-time physical activity and risk of type 2 diabetes: a meta-analysis of prospective cohort studies. Endocrine 2016; 52(2):226-230.

19. Costa-Júnior JM, Ferreira SM, Protzek AO, Santos GJ, Cappelli AP, Silveira LR, Zoppi C, Oliveira CAM, Boschero AC, Carneiro EM, Rezende LF. Endurance Training Inhibits Insulin Clearance and IDE Expression in Swiss Mice. PLoS One 2015; 10(3):e0118809. Prado MAMB, Francisco PMSB, Barros MBA. Diabetes em idosos: uso de medicamentos e risco de interação medicamentosa. Cien Saude Colet 2016; 21(11):34473458.

20. Prado MAMB, Francisco PMSB, Barros MBA. Diabetes em idosos: uso de medicamentos e risco de interação medicamentosa. Cien Saude Colet 2016; 21(11):3447-3458.

21. Bird SR, Hawley JA. Exercise and type 2 diabetes: New prescription for an old problem. Maturitas 2012; 72(4):311-316.

22. Little JP, Gillen JB, Percival ME, Safdar A, Tarnopolsky MA, Punthakee Z, Jung ME, Gibala MJ. Low-volume high-intensity interval training reduces hyperglycemia and increases muscle mitochondrial capacity in patients with type 2 diabetes. J Appl Physiol 2011; 111(6):1554-1560.

23. Marwick TH, Hordern MD, Miller T, Chyun DA, Bertoni AG, Blumenthal RS, Philippides G, Rocchini A, Council on Clinical Cardiology, American Heart Association Exercise, Cardiac Rehabilitation, and Prevention Committee; Council on Cardiovascular Disease in the Young; Council on Cardiovascular Nursing; Council on Nutrition, Physical Activity, and Metabolism; Interdisciplinary Council on Quality of Care and Outcomes Research. Exercise Training for Type 2 Diabetes Mellitus: Impact on Cardiovascular Risk: A Scientific Statement from the American Heart Association. Circulation 2009; 119(25):3244-3262.

24. Instituto Brasileiro de Geografia e Estatística (IBGE). Pesquisa nacional de saúde, 2013: percepção do estado de saúde, estilos de vida e doenças crônicas: Brasil, grandes regiões e unidades da Federação. Rio de Janeiro: IBGE; 2014.

25. Hill AB. The environment and disease: association or causation? J R Soc Med 2015; 108(1):32-37.

Artigo apresentado em 12/12/2018

Aprovado em 15/02/2019

Versão final apresentada em 17/02/2019 